\title{
Existence and possible roles of independent non-CpG methylation in mammalian brain
}

\section{CURRENT STATUS: POSTED}

Research Square

Jong-Hun Lee

The University of Tokyo

Yutaka Saito

National Institute of Advanced Industrial Science and Technology

Sung-Joon Park

The University of Tokyo

Kenta Nakai

the University of Tokyo

v knakai@ims.u-tokyo.ac.jpCorresponding Author

ORCiD: https://orcid.org/0000-0002-8721-8883

DOI:

10.21203/rs.2.22195/v1

SUBJECT AREAS

Epigenetics \& Genomics

KEYWORDS

Non-CpG methylation, Hidden Markov model, Neuro-epigenetics 
Abstract

Background DNA methylations at non-CpG sites ( $\mathrm{mCpHs}$ ) yield weak enrichment signals and colocalize with methylated CpGs (mCpGs). Accordingly, they are thought to represent byproducts of hyperactive methyltransferases. However, recent studies revealed that $\mathrm{mCpHs}$ are highly cell typespecific and associated with cellular functions in mammal, although their distributional and functional dependency to mCpGs remains to be elucidated.

Results Through computational analysis of a large publicly available DNA methylation dataset from human and mouse cells, we revealed that $\mathrm{mCpHs}$ tend to co-localize with $\mathrm{mCpGs}$ in pluripotent stem cells but not in brain cells. A systematic investigation using an empirical hidden Markov model confirmed that there are genomic regions where $\mathrm{CpGs}$ and $\mathrm{CpH}$ s are differentially methylated in the brain cells ( $\sim 25 \%)$. Remarkably, the regions were frequently located in putative enhancers, and $\mathrm{mCpHs}$ within the enhancers were greatly increased as brain aged. We detected 1669 hyper-mCpH enhancers functionally enriched to immune system development, aberration of which can lead to neuro-inflammation and neuro-degeneration.

Conclusions In mammalian brain cells, there are genomic regions where $\mathrm{CpH}$ alone is methylated (or de-methylated). The $\mathrm{mCpHs}$ are accumulated in enhancers as brain aged, potentially associated to neuro-inflammation and degeneration. Altogether, this study provides insight into the roles of nonCpG methylation as an epigenetic code in the genomes of mammalian brain cells.

\section{Full-text}

Due to technical limitations, full-text HTML conversion of this manuscript could not be completed. However, the manuscript can be downloaded and accessed as a PDF.

Figures 
(a)

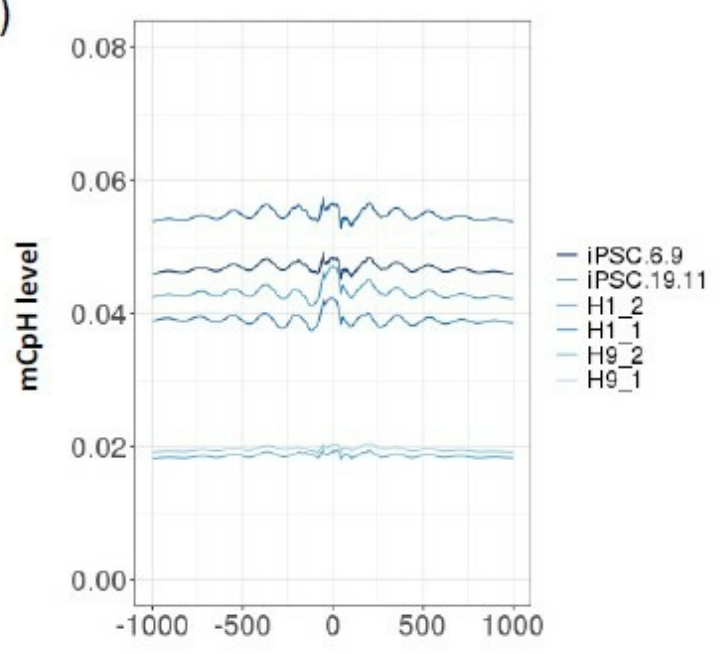

(b)

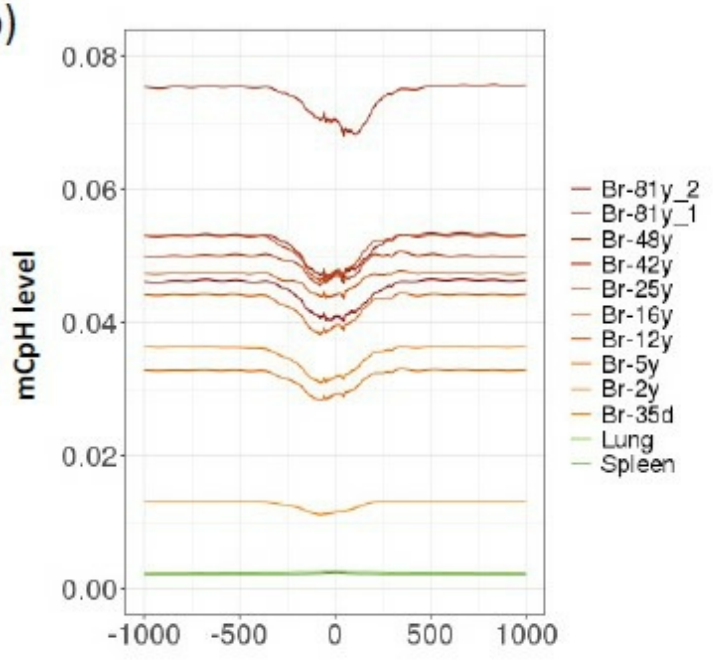

(c)

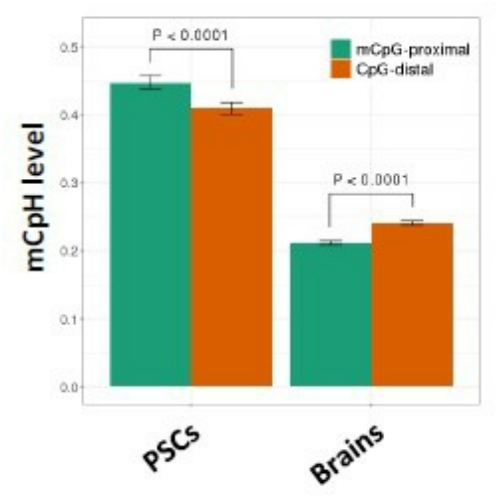

(d)
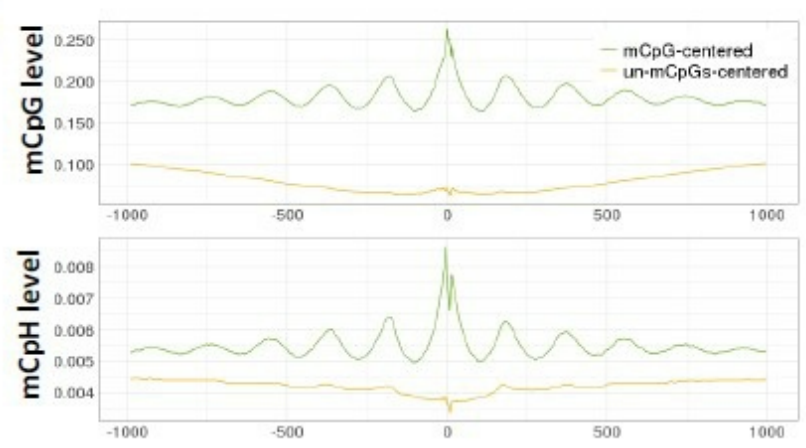

Figure 1

(a) Distribution of $\mathrm{mCpH}$ levels around $\mathrm{mCpGs}$ in human PSCs. (b) Distribution of $\mathrm{mCpH}$ levels around mCpGs in brain and control tissues. Numbers following underscores identify biological replicates. (c) Difference in $\mathrm{mCpH}$ levels at $\mathrm{mCpG}$-proximal $(< \pm 100 \mathrm{bp})$ and $\mathrm{CpG}$ distal ( $> \pm 100 \mathrm{bp}$ ) $\mathrm{CpH}$ sites. Beta values were used as average methylation levels, and Pvalue was calculated by the Wilcoxon signed-rank test. (d) Distribution of methylation levels around mCpGs and unmethylated CpGs in DNMT1-knockout mouse ESCs. mCpG, methylated CpG; mCpH, methylated non-CpG; PSC, pluripotent stem cell; Br, brain; y, year; d, day. 
(a)

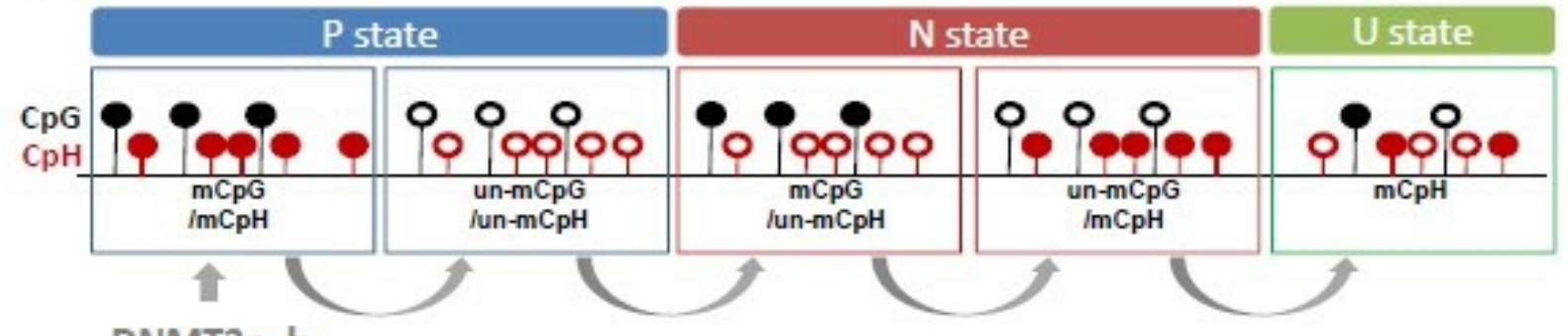

DNMT3a, b

(b)

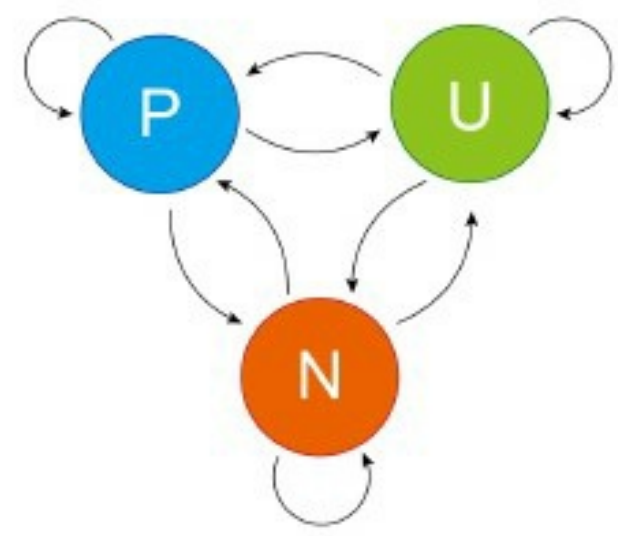

(c)

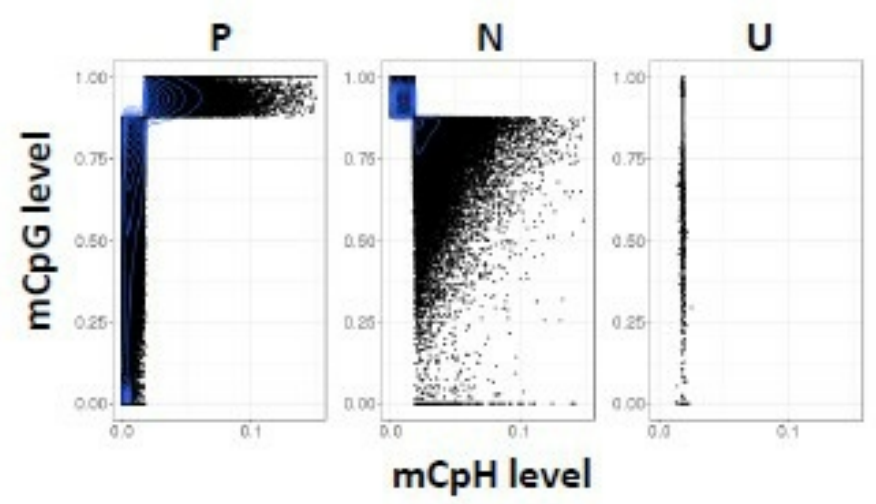

(d)

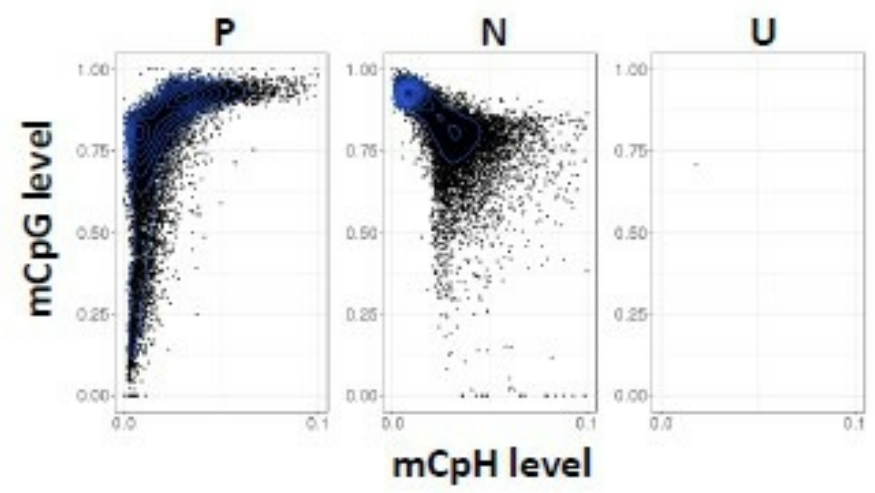

Figure 2

(a) Schematic representation of the three states of the HMM. In the P-state, either both 
CpGs and $\mathrm{CpHs}$ are methylated or neither is methylated, resulting in a positive correlation between the $\mathrm{mCpG}$ and $\mathrm{mCpH}$ levels. In the $\mathrm{N}$-state, only $\mathrm{CpGs}$ or $\mathrm{CpHs}$ are methylated, resulting in a negative correlation between the levels. In the U-state, the levels are not correlated. The bin size was set to 180 bp because DNMT3a and DNMT3b methylate DNA strands with a 180 bp periodicity. N-state bins are considered as CpG-CpH differentially methylated regions ( $\mathrm{CpG}-\mathrm{CpH}$ DMRs). (b) Schematic diagram of the state transition in the HMM. The transition probability was estimated using the EM algorithm. (c) Distribution of methylation levels of each bin assigned to one of the three states, using the highest emission probability. (d) Distribution of methylation levels of each bin assigned to one of the three states, using the Viterbi algorithm. (c) and (d) used $180 \mathrm{bp}$ bins in chromosome 19 of sample Br-81y_1. 
(a)

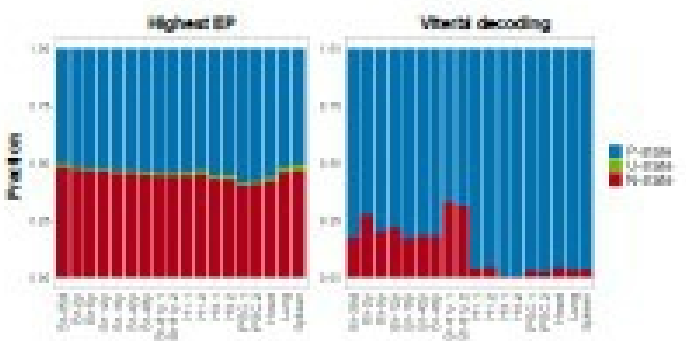

(b)

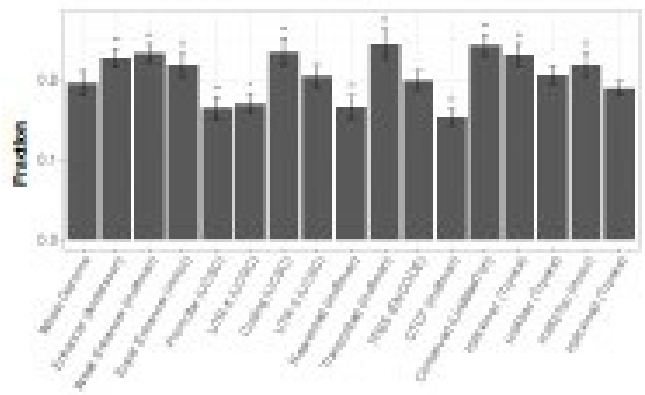

(c)

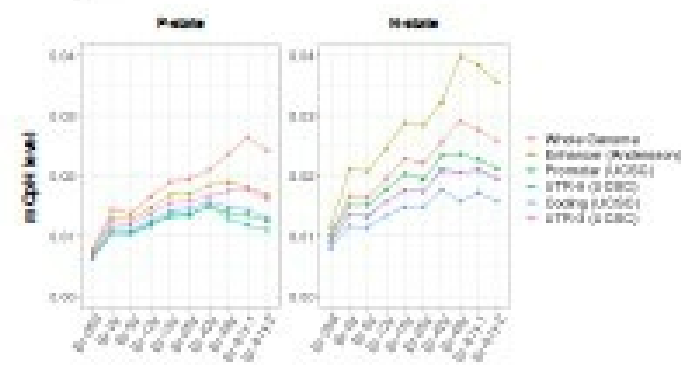

(d)

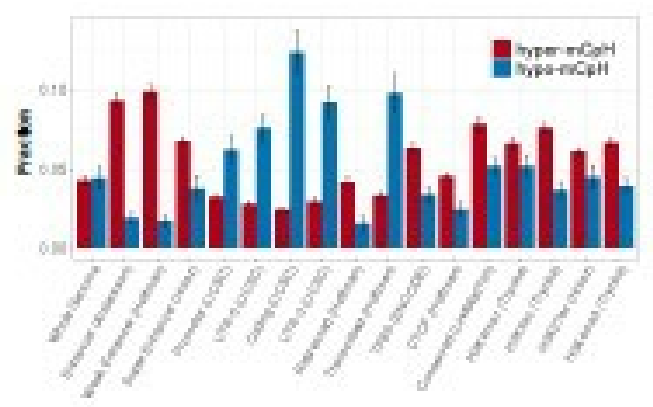

Figure 3

(a) Fraction of the $\mathrm{P}_{-}, \mathrm{N}-$, and $\mathrm{U}$-state regions detected by the highest emission probability or by the Viterbi algorithm. EP, emission probability. (b) Fraction of whole genome and of the known genomic elements overlapped with DMRs. The P-value of Student's t-test between Nstate regions in the whole genome, and in each genomic element within the brain samples, was marked as follows: $*,<0.01 ; * *,<0.001$. (c) Distribution of $\mathrm{mCpH}$ levels in $\mathrm{P}$ - and $\mathrm{N}$ state regions of brain cells. (d) Fraction of whole genome and of the known genomic 
elements overlapped with hyper- or hypo-mCpH-Ns.

(a)

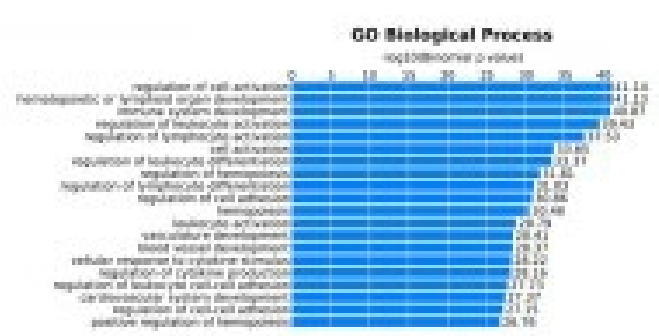

(b)
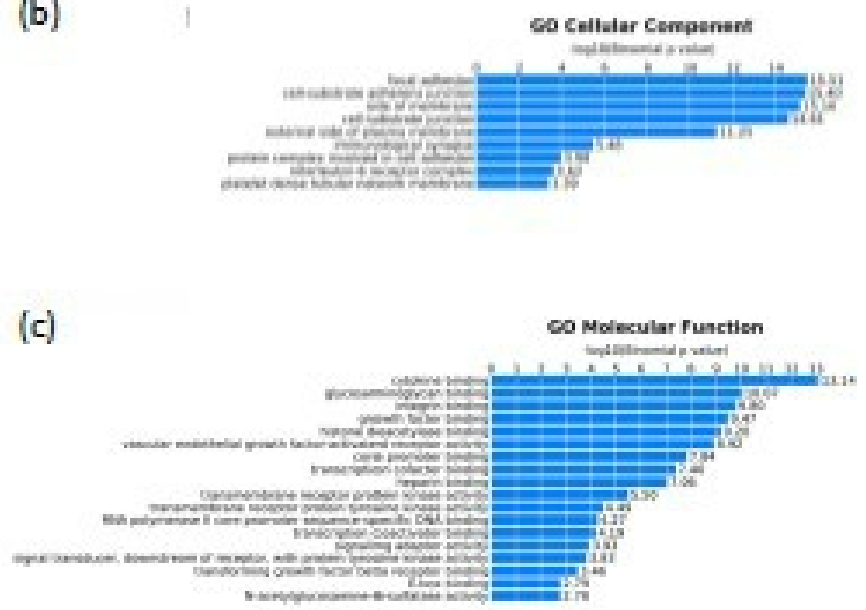

(d)

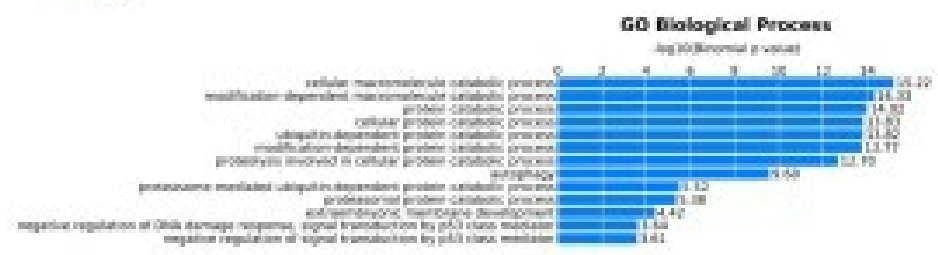

Figure 4

Results of Gene Ontology (GO) enrichment analysis of 1717 genes associated with hypermCpH-N enhancers: (a) Enriched GO biological process terms (FDR<0.05). Top 20 terms were shown. (b) Enriched GO cellular component terms. (c) Enriched GO molecular function terms. (d) Biological process terms enriched in 1467 genes associated with hypo-mCpH-N enhancers. No cellular component or molecular function terms were significantly enriched for the gene set. 


\section{Supplementary Files}

This is a list of supplementary files associated with this preprint. Click to download.

Supplementary_Figures.pdf

Supplementary_Tables.xlsx 